$\begin{gathered}\text { EPiC Series in Education Science } \\ \text { Volume 1, 2017, Pages 496-504 }\end{gathered}$
$\begin{gathered}\text { AUBEA 2017: Australasian Universities Build- } \\ \text { ing Education Association Conference 2017 }\end{gathered}$

\title{
Overview and Analysis of Digital Technologies for Construction Safety Management
}

\author{
B. H. W. Guo ${ }^{1}$, E. Scheepbouwer ${ }^{2}$, T. W. Yiu ${ }^{3}$, and V. A. González ${ }^{4}$ \\ ${ }^{1,2}$ University of Canterbury, Christchurch, New Zealand \\ ${ }^{3,4}$ University of Auckland, Auckland, New Zealand \\ brian.guo@canterbury.ac.nz, eric.scheepbouwer@canterbury.ac.nz, \\ $\mathrm{k} \cdot \mathrm{yiu} @ a u c k l$ and.ac.nz, v.gonzalez@auckland.ac.nz
}

\begin{abstract}
Digital technologies are increasingly used to support safety management in the construction industry. Previous efforts were made to identify digital technologies for safety in the construction industry. However, limited research has been done to conceptualize the roles played by digital technologies in safety management and accident prevention. This paper surveys state-of-the-art research between 2000 and 2016 in order to categorize digital technologies for construction safety, identify research trend, and analyze their roles in accident prevention. The research employs a systematic process to review the existing literature on digital technologies in the area of construction safety. Five academic databases, Science Direct, Taylor \& Francis, the ASCE Library, Engineering village, and Web of Science, were selected for the survey due to the comprehensive coverage of relevant academic papers. The survey identified 15 digital technologies: real-time location system and proximity warning, building information modelling, augmented reality, virtual reality, game technology, e-safetymanagement-system, case-based reasoning, rule-based reasoning, motion sensor, action/object recognition, laser scanning, physiological status monitoring, virtual prototyping, geographical information system, and ubiquitous sensor network. Three emerging safety functions claimed and/or promoted by DTs were discussed: enhanced safety planning, real-time hazard management, and safety knowledge engineering. It is concluded that DTs have great potential to improve safety performance by engineering resilience and adaptiveness at the individual level, while how DTs embody safety values and how safety values in turn influence the adoption of DTs remain an open question.
\end{abstract}




\section{Introduction}

We are living in a digital age. Digital technologies (DTs) have changed the way people live, work, communicate, and learn. The past two decades have seen a growing interest among researchers in applying DTs to safety management in the construction industry. A powerful motivator is that construction safety performance has reached a plateau and that DTs have promising potential to eliminate the bottleneck. It is widely believed that DTs can improve limited human conditions and thus revolutionize traditional safety management process which is largely manual, time-consuming and error-prone.

Previous efforts were made to systematically review the technology applications for construction safety. For example, Zhou et al. (2012) reviewed two different strands of research, digital tools for managing safety through construction and design, in order to understand the relationship between digital technologies and safety performance. In addition, Zhou et al. (2013) provided a general overview of technology applications for construction safety from 1986 to 2012. Emphasis has been placed on identifying type of technology, project phase, and project type. This paper extends recent literature review on DT applications to construction safety by placing emphasis on theorizing the role of DT in accident prevention and safety management. In specific, the objectives of this paper are to (1) identify the development and applications of digital technologies to construction safety, (2) analyze the functions of digital technologies and their roles in accident prevention and safety management.

\section{Methods}

This paper uses a method of literature review adopted by Zhou et al.(2013). The method consists of three main steps: (1) literature search, (2) literature selection, and (3) literature coding.

Five academic databases, Science Direct, Taylor \& Francis, the ASCE Library, Engineering village, and Web of Science, were selected for the survey due to the comprehensive coverage of relevant peer-refereed academic papers. 656 papers were identified by the preliminary search. Search terms and results are presented in Table 1.

\begin{tabular}{llc}
\hline \multicolumn{1}{c}{ Datebase } & \multicolumn{1}{c}{ Search terms } & Results \\
\hline ASCE & "BIM" OR "rule-based reasoning" OR "case-based reasoning" & 291 \\
Library & OR "expert systems" OR "wearable technology" OR "sensing" & \\
& OR "warning" OR "virtual reality" OR "augmented reality" OR & \\
& "wireless technology" OR "geographic positioning system" OR & \\
"GPS" AND "Safety" & 52 \\
village & (((("BIM" OR "rule-based reasoning" OR "case-based & \\
& reasoning" OR "expert systems" OR "wearable technology" OR & \\
& "sensing" OR "warning" OR "virtual reality" OR "augmented \\
& reality" OR "wireless technology" OR "geographic positioning \\
& system" OR "GPS") WN TI) AND ((construction) WN TI)) \\
ANDience & TITLE-ABSTR-KEY(BIM OR building information modelling & \\
Direct & OR rule-based reasoning OR case-based reasoning OR expert \\
& systems OR wearable technology OR sensing and warning OR \\
& virtual reality OR augmented reality OR wireless technology OR & \\
& geographic positioning system OR GPS ) and (Construction & \\
& AND safety).
\end{tabular}




\begin{tabular}{lll}
\hline Taylor \& & \multicolumn{1}{c}{ [[All: "construction safety"] AND [All: "bim"]] OR [All: } & 185 \\
Francis & "rule based reasoning"] OR [All: "case-based \\
& reasoning"] OR [All: "expert systems"] OR [All: "wearable & \\
& technology"] OR [All: "sensing"] OR [All: "warning"] OR [All: & \\
& "virtual reality"] OR [All: "augmented reality"] OR [All: \\
& "wireless technology"] OR [All: "geographic positioning \\
& system"] OR [All: "gps"] AND [Publication Date: & \\
& $(01 / 01 / 2000$ TO 12/31/2016)] & \\
Web & TITLE: (BIM OR building information modelling OR rule- & 35 \\
Science & based reasoning OR case-based reasoning OR expert systems \\
& OR wearable technology OR sensing and warning OR virtual \\
& reality OR augmented reality OR wireless technology) & \\
& AND TITLE: (construction) AND TITLE: (safety $\quad$ OR \\
& accident OR hazard) \\
\hline
\end{tabular}

Table 1: Preliminary search terms and results

In the literature selection phase, all book reviews, editorials, and conference papers were excluded and only journal papers were selected. This resulted in 111 papers for further analysis. All remaining papers were coded according to (1) title, (2) publication year, (3) digital technology, (4) journal title, and (5) country or region (all authors were counted).

\section{Results}

Results are presented as follows in terms of journal title, country or region, publication year, type of DT, and annual distribution of DT.

As Table 2 shows, Automation in Construction (AIC) and Journal of Computing in Civil Engineering (JCCE) cover around 59\% of the identified journal articles, with 45 and 20 papers, respectively, published between 2000 and 2016. Apart from Safety Science (SS) and Journal of Construction Engineering and Management (JCEM) which published 12 and 12 papers, respectively, other journals contain proportionally much less coverage.

\begin{tabular}{lc}
\hline \multicolumn{1}{c}{ Journal title } & $\begin{array}{c}\text { Number } \\
\text { of papers }\end{array}$ \\
\hline Automation in Construction & 45 \\
Journal of Computing in Civil Engineering & 20 \\
Safety Science & 12 \\
Journal of Construction Engineering and Management & 12 \\
Advanced Engineering Informatics & 7 \\
Construction Management and Economics & 6 \\
KSCE Journal of Civil Engineering & 2 \\
Journal of Safety Research & 1 \\
Expert Systems with Applications & 1 \\
Journal of Professional Issues in Engineering Education and Practice & 1 \\
Applied Mechanics and Materials & 1 \\
Safety and Health at Work & 1 \\
International Journal of Project Management & 1 \\
Accident Analysis and Prevention & 1 \\
\hline
\end{tabular}

Table 2: Journal title and number of reviewed papers 
As shown in Figure 1, the reviewed 111 papers are from 20 countries/regions. Among these, authors from USA and China participated in around $45 \%$ of all reviewed papers.

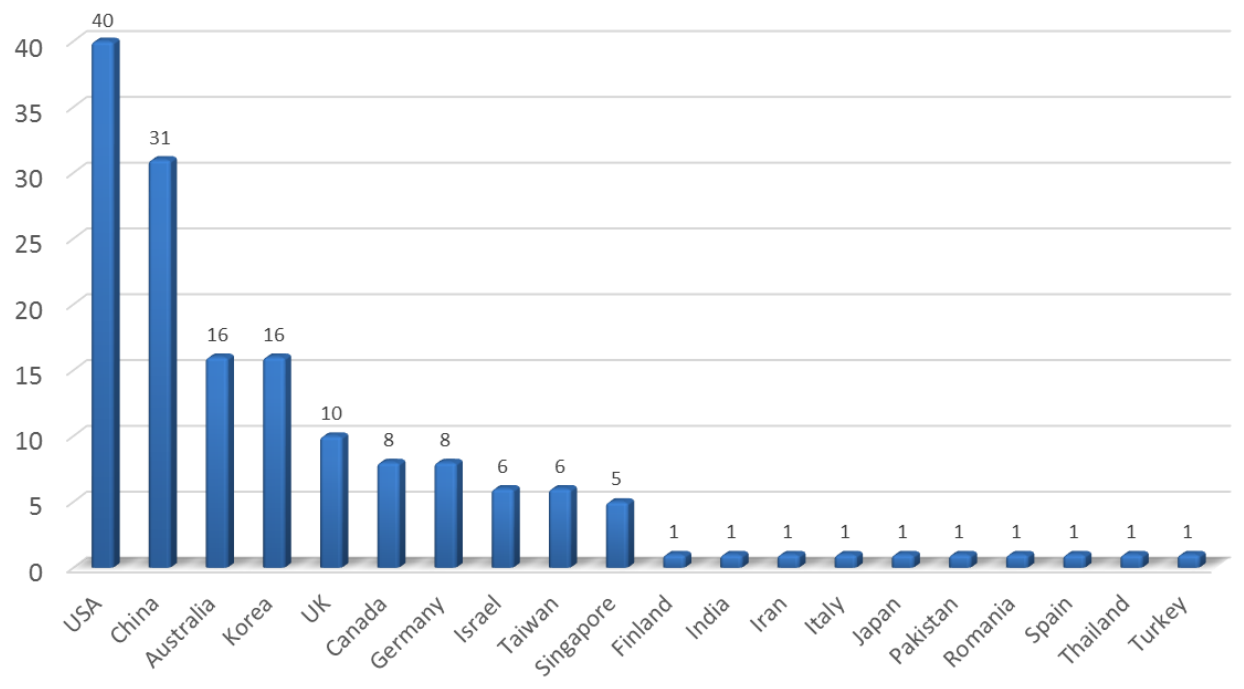

Figure 1: Geographical distribution of publications

There is an increasing number of papers between 2002 and 2016, as indicated in Figure 2.

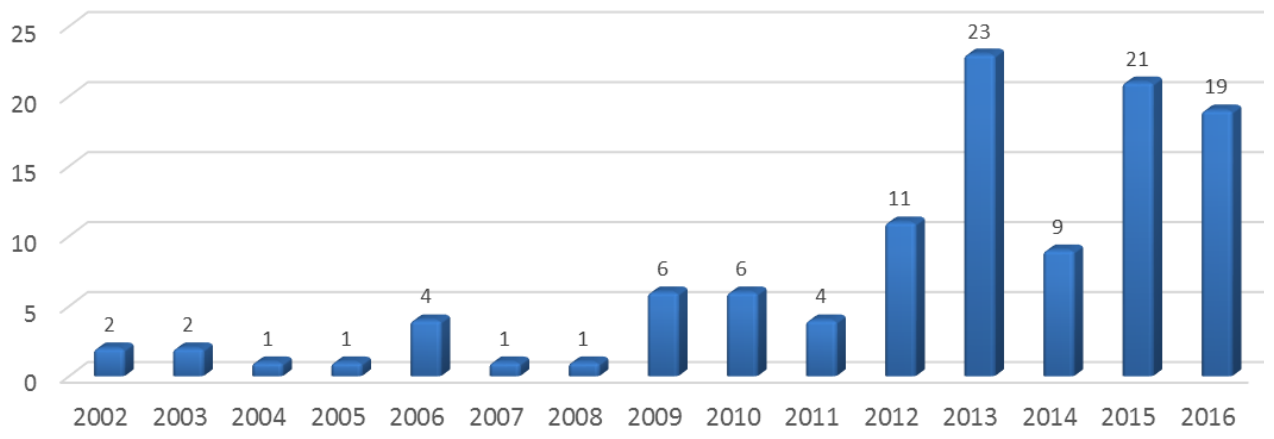

Figure 2: Annual distribution of publications from 2002 to 2016

15 DTs were identified from the literature review, including, real-time location system and proximity warning (RTLS-PW), building information modelling (BIM), augmented reality (AR), virtual reality (VR), game technology(GT), e-safety-management-system (ESMS), case-based reasoning (CBR), rule-based reasoning (RBR), motion sensor (MS), action/object recognition (AR/OR), laser scanning (LS), physiological status monitoring (PSM), virtual prototyping (VP), geographical information system (GIS), and ubiquitous sensor network (USN).

RTLS-PW covers a wide range of technologies, such as Radio frequency identification (RFID), Global positioning system (GPS), Ultra-wideband (UWB), Vision analysis, Wireless local area network (WLAN), Ultrasound, and Infrared (IR), with different features, advantages, and limitations (Li et al., 2016; Wang and Razavi, 2015). The main purpose of these technologies is to track workers, materials, and equipment and provide real-time warnings when workers approach to hazardous areas (Luo et al., 2016). As suggested in Table 3, 40 reviewed papers apply the RTLS-PW and there has 
been an increasing trend in recent years. As BIM gains it popularity across the architecture, engineering and construction (AEC) industry, there has been an increasing number of papers using BIM for construction safety, with 30 papers between 2002 and 2016. AR is "an environment where data generated by a computer is inserted into the user's view of a real world scene" (Wang et al., 2013), while VR replaces the real world with a simulated one. These two exciting technologies have often been integrated with BIM, or used alone, for safety education and training (Pedro et al., 2015; Sacks et al., 2013), hazard identification (Perlman et al., 2014), and design for safety (Hadikusumo and Rowlinson, 2002). Game technology has also been applied with attempt to enhance safety training (Guo et al., 2012).

\begin{tabular}{|c|c|c|c|c|c|c|c|c|c|}
\hline DT & $2000-2009$ & 2010 & 2011 & 2012 & 2013 & 2014 & 2015 & 2016 & Total \\
\hline $\begin{array}{l}\text { RTLS- } \\
\text { PW }\end{array}$ & 5 & 3 & 2 & 3 & 8 & 2 & 7 & 10 & 40 \\
\hline BIM & 2 & 1 & 2 & 0 & 7 & 2 & 9 & 7 & 30 \\
\hline VR & 4 & 0 & 0 & 1 & 3 & 1 & 2 & 1 & 11 \\
\hline AR & 1 & 0 & 0 & 1 & 5 & 1 & 2 & 0 & 10 \\
\hline RBR & 2 & 0 & 0 & 1 & 2 & 0 & 2 & 2 & 9 \\
\hline ESMS & 2 & 0 & 0 & 0 & 2 & 1 & 1 & 0 & 6 \\
\hline CBR & 1 & 1 & 0 & 0 & 1 & 0 & 1 & 0 & 4 \\
\hline GIS & 1 & 0 & 1 & 0 & 1 & 0 & 0 & 1 & 4 \\
\hline GT & 0 & 0 & 0 & 2 & 1 & 0 & 0 & 0 & 3 \\
\hline AR/OR & 0 & 0 & 0 & 2 & 0 & 0 & 1 & 0 & 3 \\
\hline MS & 0 & 0 & 0 & 1 & 1 & 1 & 0 & 0 & 3 \\
\hline VP & 0 & 0 & 0 & 1 & 1 & 0 & 0 & 0 & 2 \\
\hline LS & 0 & 0 & 0 & 0 & 0 & 1 & 1 & 0 & 2 \\
\hline PSM & 0 & 0 & 0 & 0 & 1 & 0 & 0 & 0 & 1 \\
\hline USN & 0 & 0 & 0 & 1 & 0 & 0 & 0 & 0 & 1 \\
\hline
\end{tabular}

Table 3: Annual distribution of DTs

\section{Discussion}

This section discusses key functions provided by the DTs. Due to the word limit, only three emerging functions and important research areas which are facilitated by DTs are discussed: enhanced safety planning, real-time hazard management, and safety knowledge engineering.

\subsection{Enhanced safety planning}

Safety planning is a core element of safety management system. Traditional safety planning is mainly concerned with identifying and managing hazards in the construction phase. The process has been time-consuming and error-prone due to the dynamic nature of construction site (Melzner et al., 2013). Another major limitation is that it is usually carried out only during the construction and it is often ignored in design (Toh et al., 2016). DTs, such as BIM, VR, and AR, can provide a platform which allows safety planning to be performed during the design stage. Safety-related design deficiencies, inappropriate work schedule, and hazardous materials can be "designed out" based on the communication among architects, engineers, and contractors. Such communication can be enhanced by visualization and simulation. Another major benefit of visualization and simulation is that they can identify and minimize the discrepancy between work as planned and work as done. In order to save time and reduce errors, automatic hazard checking rules have been designed and 
implemented into BIM (Zhang et al., 2015b; Zhang et al., 2013). This has promising potential to ease the tension between production and safety.

\subsection{Real-time hazard management}

In recent years, especially after 2010 , increasing efforts have been made to apply real-time locating and proximity warning technologies to construction safety (Carbonari et al., 2011; Cheng et al., 2012; Cheng and Teizer, 2013; Chi and Caldas, 2011; Ding et al., 2013; Golovina et al., 2016; Li et al., 2015; Luo et al., 2016; Teizer et al., 2010; Yi et al., 2016). An exciting benefit of the technologies is that they can track the location of both workers, materials, and equipment and provide proactive warnings in real-time if workers are in dangerous zones.

This function is significant because of the fact that construction is highly dynamic in nature and that hazards emerge due to the dynamics. This poses a huge threat to workers who have to manage the dynamics constantly. Traditionally, workers are notified about involved hazards before they perform a task. They have to rely on their own experience and skills to manage both identified and unidentified hazards by utilizing their safety knowledge and adjusting their behavior. However, such an ability is highly subject to human and contextual factors, such as safety awareness, safety motivation, work pressure, and peer pressure. This means that workers' situational awareness is unstable in terms of where they are and whether anything/anyone around them is a hazard to their safety. Accidents are likely to occur when workers are exposed in imminent and unidentified hazards, and, at the same time, their situational awareness is at a low point.

From this perspective, the technologies have potential to revolutionize traditional hazard identification activities. Real-time data and proactive warning enable workers to identify emerged hazards and make informative and safe decisions. A certain level of situational awareness can be automatically created and maintained. A loop safety monitoring and controlling can be bridged so that hazards can be either eliminated or isolated. Thanks to the technologies, situational awareness can even be developed in machines and equipment (e.g., trucks and cranes) (Cheng and Teizer, 2012; Teizer et al., 2010). These machines and equipment are able to sense their surroundings and make safe and "intelligent" decisions. From this perspective, the technologies are able to enhance human conditions and capabilities and thus reduce human error on construction sites.

\subsection{Safety knowledge engineering}

Another important advancement promoted by, and in turn facilitates, DTs is ontology. Over the past five years, a number of safety-related ontologies were developed in the construction industry to formalize different domain knowledge such as construction safety in general (Zhang et al., 2015a) and active fall protection system design in specific (Guo and Goh, 2017). As a foundation of knowledge engineering, these ontologies represent pioneering efforts to engineer safety knowledge and apply safety science to the construction industry. Fox (2011) defined knowledge engineering as "the engineering discipline that involves integrating knowledge into computer systems in order to solve complex problems normally requiring a high level of human expertise". Safety knowledge engineering is a significant research area because a proportion of safety-oriented DTs would depend heavily on formalized knowledge and knowledge engineering. If formalized safety knowledge is deficient, these DTs would add little value.

Safety-related ontologies formalize empirical experience, standards, regulations, and bestpractices and then facilitate the development of production rules (e.g., JESS rules and SWRL rules). These rules enable automated reasoning and problem solving. Thus, implementing relevant rules in DT platforms like BIM and Protégé allows one to undertake automatic safety management practices such as Job Hazard Analysis (JHA) (Wang and Boukamp, 2011; Zhang et al., 2015b) and safety 
checking (Lu et al., 2015; Zhang et al., 2013). These developments can potentially help people and company that have inadequate safety management capability.

\section{Conclusions}

This paper aims to (1) identify the development and applications of digital technologies to construction safety, (2) analyze the functions of digital technologies and their roles in accident prevention and safety management. In total, 15 DTs were identified by the survey, including real-time location system and proximity warning, building information modelling, augmented reality, virtual reality, game technology, e-safety-management-system, case-based reasoning, rule-based reasoning, motion sensor, action/object recognition, laser scanning, physiological status monitoring, virtual prototyping, geographical information system, and ubiquitous sensor network.

The survey reveals that a vast majority of papers focus on system design and development, field experiment, and test. Little research has been done to investigate technology adoption and implementation across construction firms and projects. As such, there is lack of solid evidence that DTs have actually improved safety performance.

Nevertheless, it is concluded that DTs have demonstrated great potential to improve safety performance by enhancing safety planning, allowing real-time hazard management, and promoting safety knowledge engineering. It is clear that if the DTs are adopted and implemented, they can enhance adaptability at the individual level. The attribute is an important asset to site safety, given the fact that workers have to manage both identified and unidentified hazards in a highly dynamic environment. This means they have to manage discrepancies between work-as-imagined and work-asdone. This has been challenging due to limited human conditions and a low level of safety knowledge. DTs are useful as they, if adopted, can enhance traditional safety training, and, more importantly, create and maintain a passive but real-time situational awareness so that workers keep "connecting" with their surroundings. From a Resilience Engineering perspective, the adaptability at the individual level constitutes an essential component of resilience of the system (a construction company or a project) as a whole. The adaptability, resilience as well, improves the system's ability to deal with pressures and changes (e.g., work pressure, peer pressure, and temporary workers).

However, over-relying on DTs may be dangerous. Given lack of understanding how DTs affect worker's safety motivation and awareness, it may be an oversimplified view that DTs will be able to deliver all their promises once they are implemented in real construction projects. In addition, it is possible that there could be a collision between DTs and safety values. How DTs embody safety values and how safety values in turn influence the adoption of DTs remain an open question. Future research efforts should be made to understand DTs in a wide organizational context and theorize the roles played by DTs in a valid safety model.

\section{Reference}

Carbonari, A., Giretti, A., Naticchia, B., 2011. A proactive system for real-time safety management in construction sites. Automation in Construction 20, 686-698.

Cheng, T., Migliaccio, G.C., Teizer, J., Gatti, U.C., 2012. Data fusion of real-time location sensing and physiological status monitoring for ergonomics analysis of construction workers. Journal of Computing in Civil Engineering 27, 320-335. 
Cheng, T., Teizer, J., 2012. Modeling tower crane operator visibility to minimize the risk of limited situational awareness. Journal of Computing in Civil Engineering 28, 04014004.

Cheng, T., Teizer, J., 2013. Real-time resource location data collection and visualization technology for construction safety and activity monitoring applications. Automation in Construction 34, 3-15.

Chi, S., Caldas, C.H., 2011. Image-based safety assessment: automated spatial safety risk identification of earthmoving and surface mining activities. Journal of Construction Engineering and Management 138, 341-351.

Ding, L., Zhou, C., Deng, Q., Luo, H., Ye, X., Ni, Y., Guo, P., 2013. Real-time safety early warning system for cross passage construction in Yangtze Riverbed Metro Tunnel based on the internet of things. Automation in Construction 36, 25-37.

Fox, J., 2011. Formalizing knowledge and expertise: where have we been and where are we going? The Knowledge Engineering Review 26, 5-10.

Golovina, O., Teizer, J., Pradhananga, N., 2016. Heat map generation for predictive safety planning: Preventing struck-by and near miss interactions between workers-on-foot and construction equipment. Automation in Construction 71, 99-115.

Guo, B.H.W., Goh, Y.M., 2017. Ontology for design of active fall protection systems. Automation in Construction.

Guo, H., Li, H., Chan, G., Skitmore, M., 2012. Using game technologies to improve the safety of construction plant operations. Accident Analysis \& Prevention 48, 204-213.

Hadikusumo, B., Rowlinson, S., 2002. Integration of virtually real construction model and design-forsafety-process database. Automation in Construction 11, 501-509.

Li, H., Chan, G., Huang, T., Skitmore, M., Tao, T.Y., Luo, E., Chung, J., Chan, X., Li, Y., 2015. Chirp-spread-spectrum-based real time location system for construction safety management: A case study. Automation in Construction 55, 58-65.

Li, H., Chan, G., Wong, J.K.W., Skitmore, M., 2016. Real-time locating systems applications in construction. Automation in Construction 63, 37-47.

Lu, Y., Li, Q., Zhou, Z., Deng, Y., 2015. Ontology-based knowledge modeling for automated construction safety checking. Safety Science 79, 11-18.

Luo, X., Li, H., Huang, T., Rose, T., 2016. A field experiment of workers' responses to proximity warnings of static safety hazards on construction sites. Safety Science 84, 216-224.

Melzner, J., Zhang, S., Teizer, J., Bargstädt, H.-J., 2013. A case study on automated safety compliance checking to assist fall protection design and planning in building information models. Construction Management and Economics 31, 661-674.

Pedro, A., Le, Q.T., Park, C.S., 2015. Framework for Integrating Safety into Construction Methods Education through Interactive Virtual Reality. Journal of Professional Issues in Engineering Education and Practice 142, 04015011.

Perlman, A., Sacks, R., Barak, R., 2014. Hazard recognition and risk perception in construction. Safety Science 64, 22-31.

Sacks, R., Perlman, A., Barak, R., 2013. Construction safety training using immersive virtual reality. Construction Management and Economics 31, 1005-1017.

Teizer, J., Allread, B.S., Fullerton, C.E., Hinze, J., 2010. Autonomous pro-active real-time construction worker and equipment operator proximity safety alert system. Automation in Construction 19, 630-640.

Toh, Y.Z., Goh, Y.M., Guo, B.H., 2016. Knowledge, Attitude, and Practice of Design for Safety: Multiple Stakeholders in the Singapore Construction Industry. Journal of Construction Engineering and Management, 04016131.

Wang, H.-H., Boukamp, F., 2011. Ontology-based representation and reasoning framework for supporting job hazard analysis. Journal of Computing in Civil Engineering 25, 442-456.

Wang, J., Razavi, S.N., 2015. Low false alarm rate model for unsafe-proximity detection in construction. Journal of Computing in Civil Engineering 30, 04015005. 
Wang, X., Love, P.E., Kim, M.J., Park, C.-S., Sing, C.-P., Hou, L., 2013. A conceptual framework for integrating building information modeling with augmented reality. Automation in Construction 34, 37-44.

Yi, W., Chan, A.P., Wang, X., Wang, J., 2016. Development of an early-warning system for site work in hot and humid environments: A case study. Automation in Construction 62, 101-113.

Zhang, S., Boukamp, F., Teizer, J., 2015a. Ontology-based semantic modeling of construction safety knowledge: Towards automated safety planning for job hazard analysis (JHA). Automation in Construction 52, 29-41.

Zhang, S., Sulankivi, K., Kiviniemi, M., Romo, I., Eastman, C.M., Teizer, J., 2015b. BIM-based fall hazard identification and prevention in construction safety planning. Safety Science 72, 31-45.

Zhang, S., Teizer, J., Lee, J.-K., Eastman, C.M., Venugopal, M., 2013. Building information modeling (BIM) and safety: Automatic safety checking of construction models and schedules. Automation in Construction 29, 183-195.

Zhou, W., Whyte, J., Sacks, R., 2012. Construction safety and digital design: A review. Automation in construction 22, 102-111.

Zhou, Z., Irizarry, J., Li, Q., 2013. Applying advanced technology to improve safety management in the construction industry: a literature review. Construction Management and Economics 31, 606-622. 